IN BRIEF

\title{
$\Rightarrow$ THERAPY
}

The ATTRACTION of nivolumab for gastric and gastro-oesophageal junction cancer

Phase III trial results reported in The Lancet indicate that immune checkpoint inhibition with nivolumab (a monoclonal antibody against PD1) could be a new treatment option for advanced gastric or gastro-oesophageal junction cancer. In the ATTRACTION-2 study, 493 patients with advanced gastric or gastro-oesophageal junction cancer who were refractory to, or intolerant of, two or more previous chemotherapy regimens were enrolled from across 49 clinical centres in Japan, South Korea and Taiwan. Patients were then randomly assigned to receive either nivolumab $(n=330)$ or placebo $(n=193)$. Median overall survival ( 5.26 months versus 4.14 months; $P<0.0001$ ) and 12 -month overall survival rates $(26.2 \%$ versus $10.9 \%$ ) were higher in the nivolumab group than in the placebo group.

ORIGINAL ARTICLE Kang, Y.-K. et al. Nivolumab in patients with advanced gastric or gastro-oesophageal junction cancer refractory to, or intolerant of, at least two previous chemotherapy regimens (ONO-4538-12, ATTRACTION-2): a randomised, double-blind, placebo-controlled, phase 3 trial. Lancet http://dx.doi.org/10.1016/S0140-6736(17)31827-5 (2017)

\section{$\Rightarrow$ GUT MICROBIOTA}

\section{Oral microbiome could provide clues to CRC}

Flemer et al. profiled the microbiota in oral swabs, colonic mucosa samples and stool from individuals with either colorectal cancer (CRC; $n=99)$, colorectal polyps $(n=32)$ or healthy individuals as controls $(n=103)$. They found that several oral taxa (such as Streptococcus and Prevotella spp.) were differentially abundant in CRC versus controls. Moreover, they developed a classification model based on oral swab microbiota that distinguished individuals with CRC or polyps from controls (sensitivity: 53\% CRC, 67\% polyps; specificity: 96\%). Importantly, when data from both faecal and oral swab microbiota were considered in this model, the sensitivity increased to $76 \%$ for CRC and $88 \%$ for polyps, respectively. Finally, high abundance of Lachnospiraceae was negatively correlated with a Western diet and colonization of colonic tissue with oral bacteria (including oral pathogens associated with CRC), hinting at a possible protective role for certain microbiota types. The authors postulate that analysing the oral microbiome could be an alternative method to screening for CRC.

ORIGINAL ARTICLE Flemer, B. et al. The oral microbiota in colorectal cancer is distinctive and predictive. Gut http://dx.doi.org/10.1136/gutjnl-2017-314814 (2017)

\section{$\Rightarrow$ IBD}

\section{The changing epidemiology of IBD}

A new study reports on the changing burden of IBD, with accelerating incidence in newly industrialized countries and a high burden in Western countries, although the incidence is stabilizing and even decreasing in some of these regions. This new systematic review examined population-based studies published from 1990 to 2016 that reported the incidence or prevalence of either Crohn's disease or ulcerative colitis, including 147 studies in the systematic review. The highest reported prevalence of IBD was in Europe and North America, with prevalence exceeding $0.3 \%$ in North America, Oceania and many European countries. Since 1990, the incidence in newly industrialized countries (in Africa, Asia and South America) has been increasing.

ORIGINAL ARTICLE Ng, S. C. et al. Worldwide incidence and prevalence of inflammatory bowel disease in the 21st century: a systematic review of population-based studies. Lancet http://dx.doi.org/10.1016/S0140-6736(17)32448-0 (2017) 